\title{
MAGNETIC FLUID-BASED SQUEEZE FILM BETWEEN CURVED POROUS ANNULAR PLATES CONSIDERING THE ROTATION OF MAGNETIC PARTICLES AND SLIP VELOCITY
}

\author{
Niru C. Patel, Jimit R. Patel* \\ Department of Mathematical Sciences, P. D. Patel Institute of Applied Sciences, CHARUSAT, \\ Changa-388 421, Anand, Gujarat, India \\ E-mail: patel.jimitphdmarch2013@gmail.com \\ *corresponding author
}

\begin{abstract}
The ferrofluid flow model of the Shliomis and continuity equation for the film and porous interface with the theory of Darcy, the modified Reynolds equation for ferrofluid squeeze film between curved annular plates are discussed with the impact of the rotation of Ferro-particles and slip velocity at the boundary. Beavers and Joseph's slip conditions are adopted to study the impact of slip velocity. The generalized non-dimensional pressure equation is derived and expression for dimensionless load-carrying capacity is obtained for the same. The graphical representation suggests that the bearing's performance enhances due to ferrofluid, considering the appropriate values of parameters for slip velocity and porosity.
\end{abstract}

Keywords: Shliomis' Magnetic fluid flow model, annular plates, porosity, slip velocity, load carrying capacity (LCC).

\section{Introduction}

In bearings, annular plates are one of the most important structural components used in industrial applications and engineering fields. They are broadly used in the production of aircraft, ships, automobiles and other vehicles. The squeezing action of the approaching surfaces causes fluid to flow toward less constrained surroundings.

A ferrofluid is synthesized in the lab and it represents a liquid that becomes strongly magnetized in the presence of a magnetic field, with friction-reducing capabilities. In recent years, ferrofluid has had many industrial applications, because of the increased use of liquid metal lubricants at high temperatures. The slip velocity depends mainly on the difference in density between the two fluids and their holdups.

In the last three decades, several researchers have analyzed the effect of magnetic fluids in various bearings with squeeze film phenomena such as Kumar et al. (1992) in spherical and conical bearings, cylindrical rollers by Sinha et al. (1993), Lin (2001) for parallel annular disks, Shah and Bhat (2005) in an infinitely long journal bearing, Shah and Bhat (2004) for annular plates, Patel and Deheri (2013) in circular plates, parallel plate rough slider bearing by Patel and Deheri (2016), Hanumagowda et al. (2018) in annular plates and short bearing by Munshi et al. 
(2019). They suggested that the negative effect can be easily nullified by the positive impact of the squeeze film occurrences and ferrofluid. It improved the MHD squeeze film characteristics of the system.

As per the literature, a porous medium is a well-known phenomenon to improve bearing systems' effect. Many authors dealt with the performance of porous medium in various magnetic fluid lubricated bearing systems: Agrawal (1986) in inclined slider bearing, Bhat and Deheri (1991) for composite slider bearing, annular discs by Bhat and Deheri (1991), Bhat and Deheri (1993) for circular discs, Shah and Bhat (2000) in circular plates, annular plates by Shah et al. (2002), Patel and Deheri (2014) in curved circular plates, Deheri et al. (2016) convex pad slider bearing and Vasanth et al. (2018) for the annular plate. It was examined that the load-bearing capacity was affected by the porous medium.

Nowadays, many investigators (Bujurke et al. (2007), Shimpi and Deheri (2013), Deheri and Patel (2015), Patel and Deheri (2016) and Shah et al. (2016)) motivated to prob on annular plates due to their extensive applications in various engineering fields. They have examined that we can improve the bearing's presentation by choosing suitable parameters (for instance, slip velocity, porosity, roughness, etc.). Shah and Patel (2017) discussed the ferrofluid lubricated circular discs porous squeeze film bearings with the impact of porosity, slip velocity, anisotropic permeability and rotation. It was detected that the unchanging magnetic field did not move on the demonstrations of the bearing systems. Mishra et al. (2018) studied an inclined slider bearing considering surface roughness, porosity and magnetic field effect. It was investigated that the slip parameter's adverse effect, reported in earlier studies over load-lifting capacity, turned rather sinusoidal to be encouraging, which might be due to the shape of the variation of a Ferro-field.

In the present paper, magnetic fluid's effect with the rotation of magnetic particles and slip velocity on squeeze film between curved porous annular plates has been theoretically examined.

\section{Mathematical Formulation}

As in Figure 1, the bearing is made of two annular disks with outside radius a and inside radius $b(a>b)$.

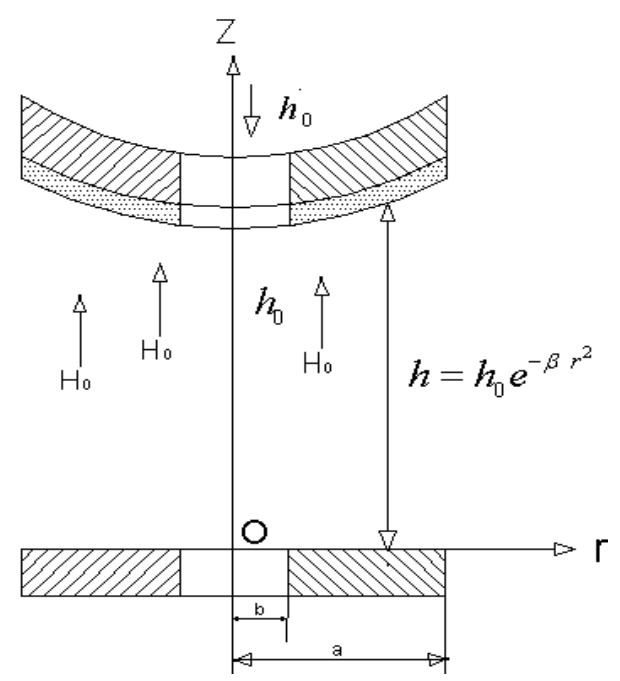

Fig. 1. Configuration of the Problem 
The film thickness $\mathrm{h}$ is considered as Murti (1975)

$$
h=h_{0} e^{-\beta r^{2}}, b \leq r \leq a
$$

Where $r, h_{0}$ and $\beta$ are radial coordinate, central film thickness and the upper plate's curvature, respectively. Two plates approach with a normal velocity $\dot{h}=\frac{d h}{d t}$.

Ferro particles can relax in two ways as the applied magnetic field changes described in Shliomis (1972) and Kumar (1989). One is by the Brownian relaxation time parameter $\tau_{B}$. Another is by the relaxation time parameter $\tau_{s}$.

Assuming steady flow, neglecting inertia and the second derivative of the internal angular momentum $\bar{s}$ without assumptions of Shukla and Kumar (1987), the equation for fluid flow of Shliomis (1972) can be governed by

$$
\begin{aligned}
-\nabla p+\eta \nabla^{2} \bar{q}+\mu_{0}(\bar{M} . \nabla) \bar{H}+\frac{1}{2 \tau_{s}} \nabla \times(\bar{S}-I \bar{\Omega})=0 \\
\bar{S}=I \bar{\Omega}+\mu_{0} \tau_{s}(\bar{M} \times \bar{H}), \\
\bar{M}=M_{0} \frac{\bar{H}}{H}+\frac{\tau_{B}}{I}(\bar{S} \times \bar{M}),
\end{aligned}
$$

Where $p$ is the pressure, $\eta$ being the viscosity of the suspension, $\mu_{0}$ stands for the permeability of free space, $\bar{H}$ is the applied magnetic field, $\bar{M}$ be the magnetization vector, $\bar{q}$ indicates the fluid velocity, $I$ is the sum of moments of inertia of the particles per unit volume, $\bar{\Omega}=(1 / 2) \nabla \times \bar{q}$, and $M_{0}$ is the equilibrium magnetization, together with continuity equation,

$$
\nabla \cdot \bar{q}=0
$$

And equations of Maxwell

$$
\begin{gathered}
\nabla \times \bar{H}=0 \\
\nabla \cdot(\bar{H}+\bar{M})=0
\end{gathered}
$$

Using equation (3), we observe that equation (2) and (4) reduces to

$$
-\nabla p+\eta \nabla^{2} \bar{q}+\mu_{0}(\bar{M} \cdot \nabla) \bar{H}+\frac{1}{2} \mu_{0} \nabla \times(\bar{M} \times \bar{H})=0
$$

and

$$
\bar{M}=M_{0} \frac{\bar{H}}{H}+\tau_{B} \bar{\Omega} \times \bar{M}-\frac{\mu_{0} \tau_{B} \tau_{s}}{I} \bar{M} \times(\bar{M} \times \bar{H})
$$

The Langevin's parameter $\xi$ is a measure of the dimensionless field strength. Neglecting the strong magnetic field $\xi>1$ and $\tau_{s}$ as per Shukla and Kumar (1987), the equation (9) can be approximated as 


$$
\bar{M}=\frac{M_{0}}{H}[\bar{H}+\bar{\tau}(\bar{\Omega} \times \bar{H})]
$$

Where

$$
\bar{\tau}=\frac{\tau_{B}}{1+\frac{\mu_{0} \tau_{B} \tau_{s}}{I} M_{0} H} M_{0}=n \mu(\operatorname{coth} \xi-1 / \xi) \text { and } H=k_{B} T \xi /\left(\mu_{0} \mu\right),
$$

with $\frac{I}{\tau_{s}}=6 \eta \varphi$ and $\tau_{B}=3 \eta V /\left(k_{B} T\right)$, where $\varphi=n V$ stands for the volume concentration of the particles, $k_{B}$ is the Boltzmann constant, $\mathrm{n}$ be the number of particles per unit volume, $T$ is the temperature and $\mu$ being the magnetic moment of a particle for spherical particles in suspension (Shliomis, 1972).

One can express $\bar{\tau}$ as

$$
\bar{\tau}=\frac{6 \eta \varphi}{n k_{B} T(1+\xi \operatorname{coth} \xi)}
$$

With uniform magnetic field $\bar{H}=\left(0,0, H_{0}\right)$ and radial velocity component $u$ in axially symmetric flow, equation (8) and (10) turns out to be

$$
\frac{\partial^{2} u}{\partial z^{2}}=\frac{1}{\eta\left(1+\frac{\mu_{0} M_{0} H_{0} \bar{\tau}}{4 \eta}\right)} \frac{d p}{d r}
$$

From equations (11-13), one obtains

$$
\frac{\partial^{2} u}{\partial z^{2}}=\frac{1}{\eta(1+\tau)} \frac{d p}{d r}
$$

Where

$$
\tau=\frac{3}{2} \varphi \frac{\xi-\tanh \xi}{\xi+\tanh \xi}
$$

Solving equation (14) under the boundary conditions with slip

$u=0$ when $z=0, u=-\frac{1}{s} \frac{\partial u}{\partial z}$ when $z=h$ with $\frac{1}{s}$ as a slip parameter.

One can derive

$$
u=-\frac{1}{\eta_{a}} \frac{z^{2}-z \operatorname{sh}(z-h)}{2(1+s h)} \frac{d p}{d r}
$$

Where $\eta_{a}=\eta(1+\tau)$

Substituting the above value of $\mathrm{u}$ in the integral form of the continuity equation 


$$
\frac{1}{r} \frac{d}{d r} \int_{0}^{h} r u d z+w_{h}-w_{0}=0
$$

yields

$$
\frac{1}{r} \frac{d}{d r}\left(\frac{h^{3} r(2+s h)}{1+s h} \frac{d p}{d r}\right)=12 \eta_{a}\left(w_{h}-w_{0}\right)
$$

With the Einstein's viscosity of the suspension $\eta=\eta_{0}\left(1+\frac{5}{2} \varphi\right)$ where the viscosity of carrier liquid $\eta_{0}$ (Shliomis, 1972)) and

$$
\eta_{a}=\eta(1+\tau)=\eta_{0}\left(1+\frac{5}{2} \varphi\right)(1+\tau)
$$

equations (17-18) take the form

$$
\frac{1}{r} \frac{d}{d r}\left(\frac{h^{3} r(2+s h)}{1+s h} \frac{d p}{d r}\right)=12 \eta_{0}\left(1+\frac{5}{2} \varphi\right)(1+\tau)\left(w_{h}-w_{0}\right)
$$

As per the discussion of Bhat (2003), the conditions for the lower surface is solid and the porous facing of thickness $H^{*}$ at the upper surface are,

$$
w_{0}=0 \text { and } w_{h}=\dot{h}_{0}-\frac{\bar{k}}{\eta_{a}} \frac{\partial P_{1}}{\partial z}
$$

Where $\dot{h}_{0}$ is the squeeze velocity, $P_{1}$ be the pressure in the porous region and permeability of the porous region $\bar{k}$.

Pressure $P_{1}$ satisfies the equation

$$
\frac{1}{r} \frac{\partial}{\partial r}\left(r \frac{\partial P_{1}}{\partial r}\right)+\frac{\partial^{2} P_{1}}{\partial z^{2}}=0
$$

Using the Morgan-Cameron approximation,

$$
\left(\frac{\partial P_{1}}{\partial z}\right)_{z=h}=\frac{H^{*}}{r} \frac{d}{d r}\left(r \frac{d p}{d r}\right)
$$

Using equation (20) and (22), equation (19) yields

$$
\frac{1}{r} \frac{d}{d r}\left(\frac{h^{3} r(2+s h)}{1+s h} \frac{d p}{d r}\right)=12 \eta_{0}\left(1+\frac{5}{2} \varphi\right)(1+\tau)\left(\dot{h}_{0}-\frac{\bar{k}}{\eta_{a}} \frac{H^{*}}{r} \frac{d}{d r}\left(r \frac{d p}{d r}\right)\right)
$$

Solving the above equation, one can find

$$
\frac{1}{r} \frac{d}{d r}\left(\left[\frac{h^{3}(2+s h)}{1+s h}+12 \bar{k} H^{*}\right] r \frac{d p}{d r}\right)=12 \eta_{0}\left(1+\frac{5}{2} \varphi\right)(1+\tau) \dot{h}_{0}
$$

Upon establishment of the non-dimensional measures: 


$$
R=\frac{r}{b}, \bar{h}=\frac{h}{h_{0}}, \bar{\beta}=\beta b^{2}, P=\frac{-h_{0}{ }^{3} p}{\eta_{0} b^{2} \dot{h_{0}}}, \bar{s}=s h_{0}, \psi=\frac{\bar{k} H^{*}}{h_{0}{ }^{3}}, k=\frac{a}{b}
$$

and use of equation (25), Equation (24) reduces to

$$
\frac{1}{R} \frac{d}{d R}\left\{\left[\frac{\bar{h}^{3}(2+\bar{s} \bar{h})}{1+\bar{s} \bar{h}}+12 \psi\right] R \frac{d P}{d R}\right\}=-12 \eta_{0}\left(1+\frac{5}{2} \varphi\right)(1+\tau)
$$

Solving Equation (26) subject to Reynolds boundary conditions for the bearing

$$
P(1)=P(k)=0
$$

One can get the dimensionless pressure $\mathrm{P}$ as

$$
P=\int_{1}^{R}\left(-\frac{6 E}{G}\right) R d R+c_{1} \int_{1}^{R}\left(\frac{1}{G R}\right) d R
$$

where

$$
E=\left(1+\frac{5}{2} \varphi\right)(1+\tau), G=\frac{\bar{h}^{3}(2+\bar{s} \bar{h})}{1+\bar{s} \bar{h}}+12 \psi, c_{1}=\frac{\int_{1}^{k} \frac{6 E R}{G} d R}{\int_{1}^{k} \frac{1}{G R} d R}
$$

The load-carrying capacity of the bearing can be found in non-dimensional form as

$$
\bar{W}=\frac{-h_{0}{ }^{3} W}{2 \pi \eta_{0} b^{4} \dot{h}_{0}}=\int_{1}^{k} R P d R=\frac{-1}{2} \int_{1}^{k} R^{2} \frac{d P}{d R} d R-\frac{1}{2}\left(\int_{1}^{k}-\frac{6 E R^{3}}{G} d R+c_{1} \int_{1}^{k} \frac{R}{G} d R\right)
$$

\section{Results and Discussion}

In section 2, we have analyzed the mathematical relations for the considered problem. Here, graphical results are discussed.

Here the equation (28) describes the non-dimensional pressure distribution, while equation (30) determines the dimensionless load carrying capacity (LCC). As compared with the conventional lubricants, this study tends to point out that the dimensionless pressure increases by

$$
\left(1+\frac{5}{2} \varphi\right) \tau \int_{R}^{1} \frac{6 R}{G} d R
$$

while the non-dimensional LCC increases by

$$
\left(1+\frac{5}{2} \varphi\right) \tau \int_{1}^{k} \frac{3 R^{3}}{G} d R
$$

due to the linearity of the magnetization parameter in both the expression.

Considering $1 / \bar{s} \rightarrow 0$ and $\psi \rightarrow 0$, one can find the effect of the Shliomis model-based magnetic fluid in squeeze film annular bearing. Further, taking $\tau \rightarrow 0$, this analysis reduces to the 
study of annular plates without lubrication (Gupta and Vora, 1980)). Moreover, this investigation is the extension of the study carried out by Shah and Bhat (2005), including the combined influence of porosity and velocity with slip at surfaces. The graphical representations of the problem are presented in Figures (2) to (10).

Figures (2) to (4) describe the variation of non-dimensional LCC concerning for Shliomis model-based magnetic parameter for different values of $\phi, \bar{\beta}, \psi$ and $1 / \bar{s}$ respectively. From Figures (2) to (4), one can find that the LCC enhances sharply with increasing magnetic parameter $\tau$. But (3) and (5) indicate that the effect of $\bar{\beta}$ and $1 / \bar{s}$ with respect to $\tau$ is not up to mark so far as the LCC is concerned.

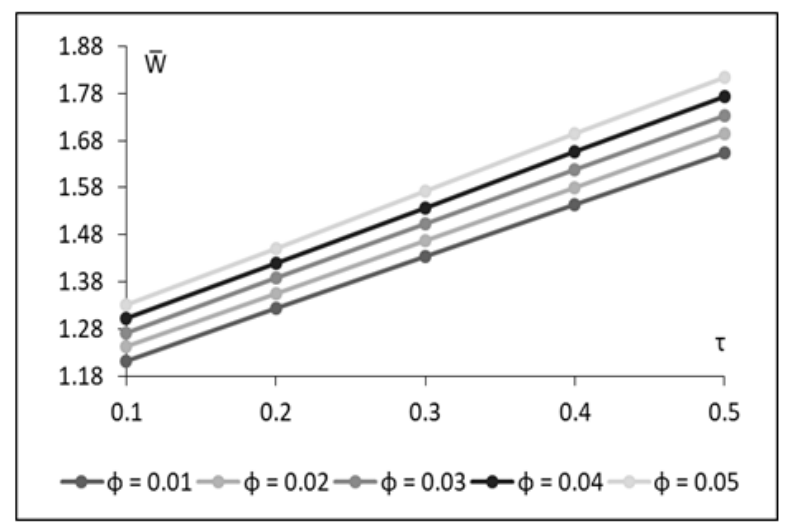

Fig. 2. Profile of LCC for $\tau$ and $\phi$ with $1 / \bar{s}=0.02, \psi=25, \bar{\beta}=1.5$.

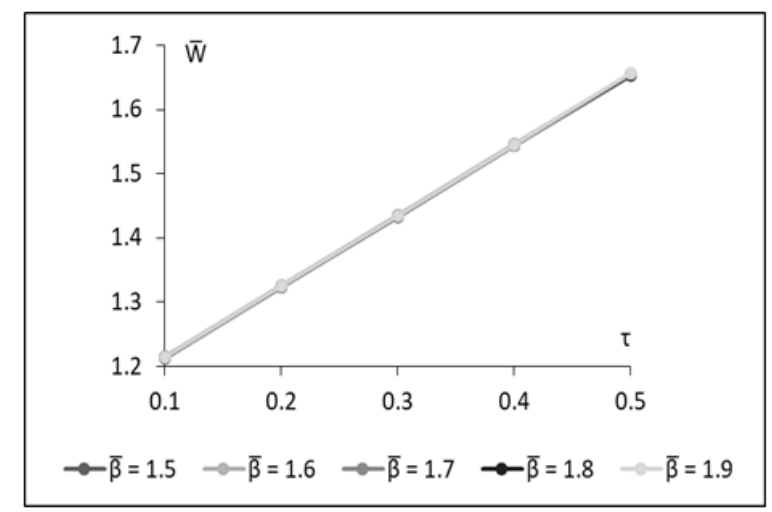

Fig. 3. Profile of LCC for $\tau$ and $\bar{\beta}$ with $1 / \bar{s}=0.02, \psi=25, \phi=0.1$. 


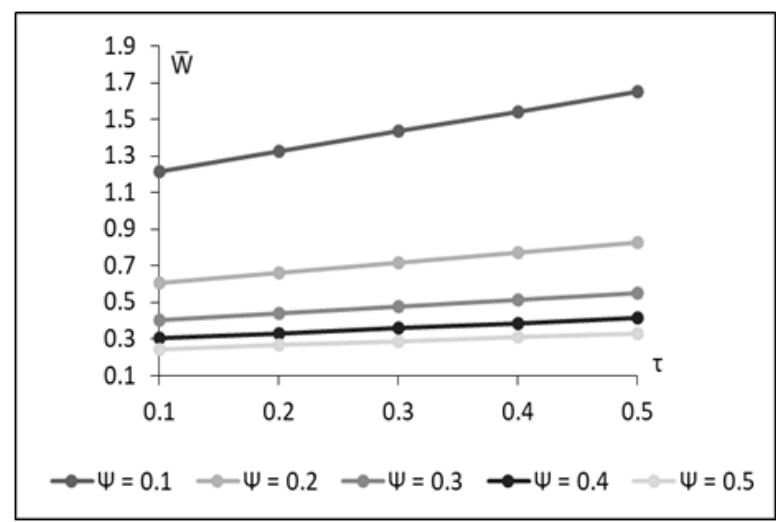

Fig. 4. Profile of LCC for $\tau$ and $\psi$ with $1 / \bar{s}=0.02 \phi=0.1, \bar{\beta}=1.5$.

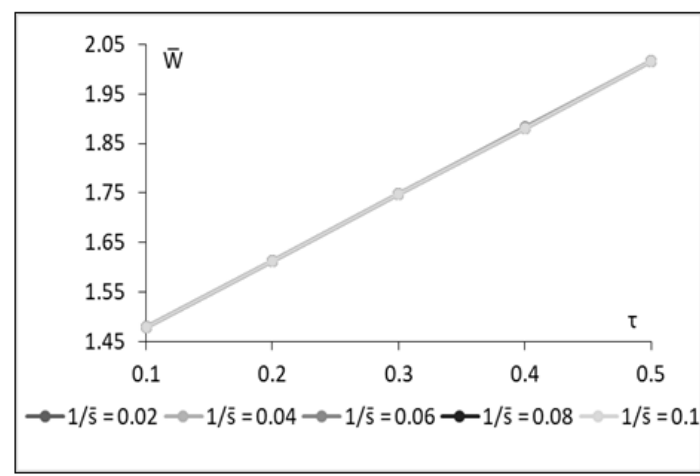

Fig. 5. Profile of LCC for $\tau$ and $1 / \bar{s}$ with $\psi=25, \phi=0.1, \bar{\beta}=1.5$.

The distribution of the LCC regarding $\phi$ for different values of $\bar{\beta}, \psi$ and $1 / \bar{s}$ is presented in Figures(6) - (8). It is noticed that $\phi$ decreases the LCC while the effect remains the same for $1 / \bar{s}$ and $\bar{\beta}$ increases LCC marginally. 


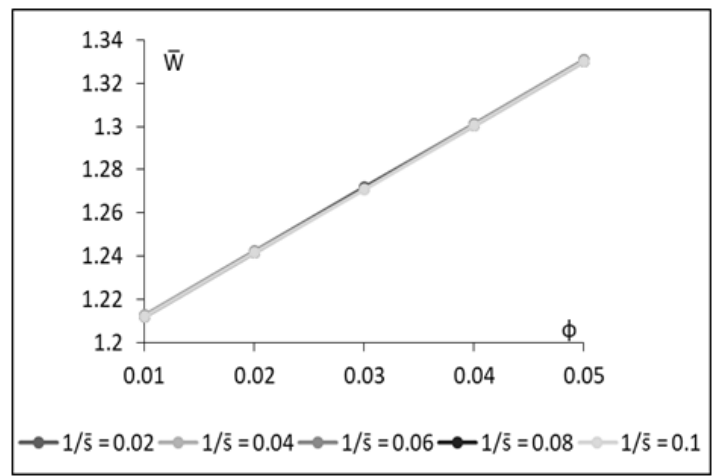

Fig. 6. Profile of LCC for $\phi$ and $1 / \bar{s}$ with $\psi=25, \tau=0.1, \bar{\beta}=1.5$.

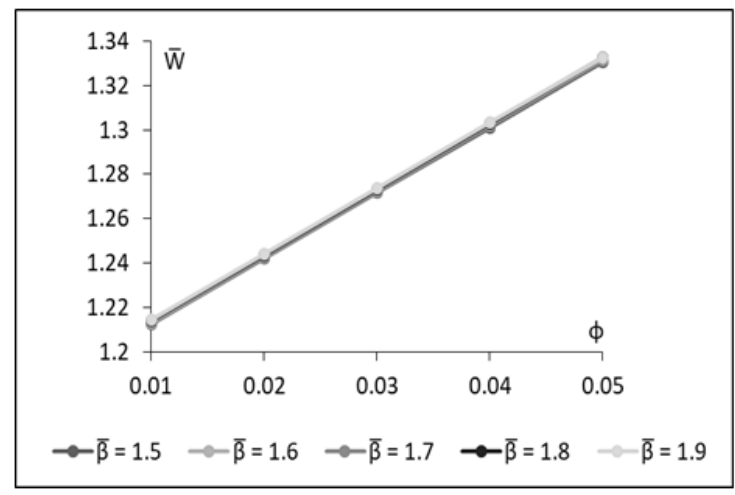

Fig. 7. Profile of LCC for $\phi$ and $\bar{\beta}$ with $\psi=25, \tau=0.1,1 / \bar{s}=0.02$.

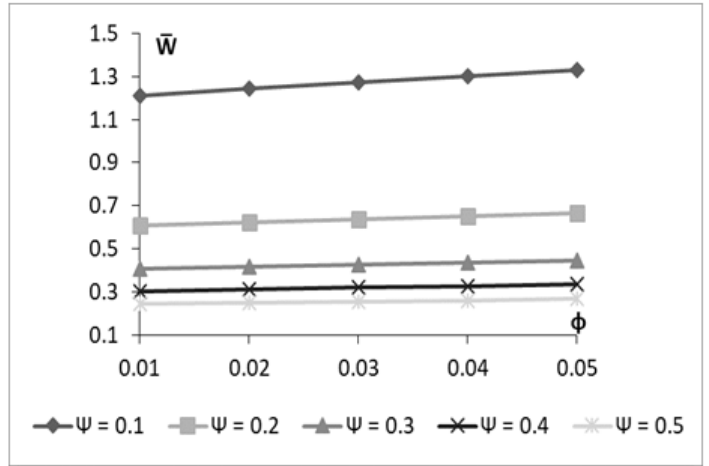

Fig. 8. Profile of LCC for $\phi$ and $\psi$ with $1 / \bar{s}=0.02, \tau=0.1, \bar{\beta}=1.5$.

From Figures (9) and (10), one can see the profile for the LCC concerning $\bar{\beta}$. for the different values of $\psi$ and $1 / \bar{s}$. The influence of LCC regarding $\bar{\beta}$ and $\psi$ has negligible effect 
as displayed in Figures (9). Although, the LCC increases with increasing the value of $\bar{\beta}$ with respect to $1 / \bar{s}$ as per figure (10).

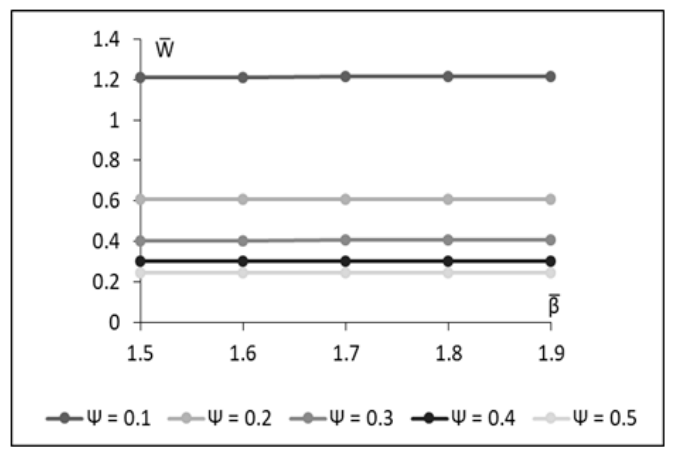

Fig. 9. Profile of LCC for $\bar{\beta}$ and $\psi$ with $1 / \bar{s}=0.02, \tau=0.1, \phi=0.1$.

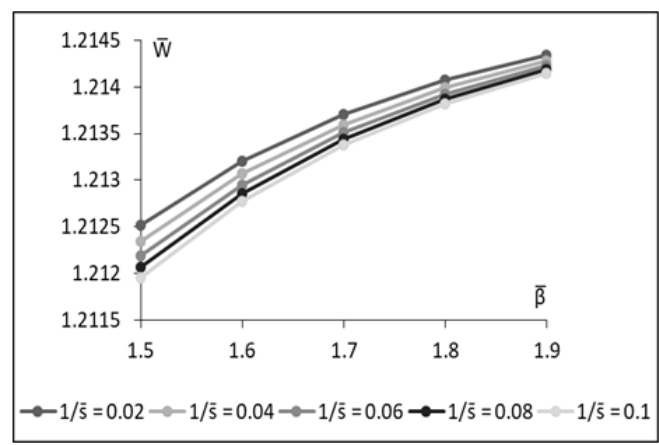

Fig. 10. Profile of LCC for $\bar{\beta}$ and $1 / \bar{s}$ with $\psi=25, \tau=0.1, \phi=0.1$

Figure (11) depicts the variation of LCC with respect to $\psi$ for various values of $1 / \bar{s}$ The rate of decrease in the LCC due to $\psi$ is quite sharp. 


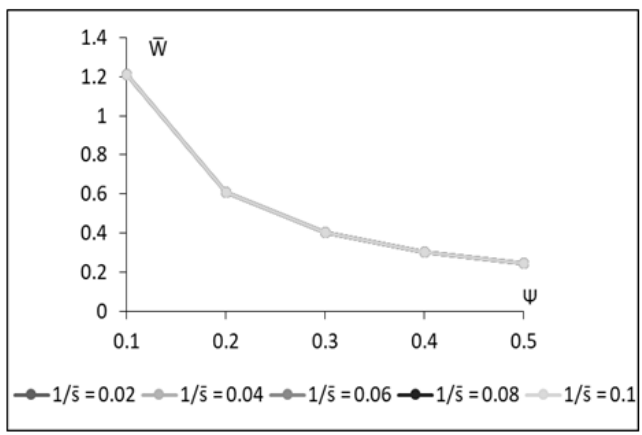

Fig. 11. Profile of LCC for $\psi$ and $1 / \bar{s}$ with $\bar{\beta}=1.5, \tau=0.1, \phi=0.1$.

From the graphical representation, one can suggest that the negative effect of slip velocity can be compensated by the magnetization parameter $\tau$ with the inclusion of the proper selection of other parameters. In general, the bearing can sustenance a load even if there is no flow.

\section{Validation}

The comparison of our study with the investigation of Shah and Bhat (2005) is discussed herein following tables to validate our research.

\begin{tabular}{|c|c|c|c|c|c|c|}
\hline & $\tau$ & 0.1 & 0.2 & 0.3 & 0.4 & 0.5 \\
\hline $\begin{array}{l}\text { Shah and Bhat } \\
(2005)\end{array}$ & \multirow{2}{*}{$\begin{array}{l}\vec{\circ} \\
0 \\
\text { II } \\
\theta\end{array}$} & 4.102285498 & 4.202341242 & 4.302396986 & 4.40245273 & 4.502508474 \\
\hline Present Study & & 6.01259653 & 6.159245226 & 6.305893922 & 6.452542617 & 6.599191313 \\
\hline $\begin{array}{l}\text { Shah and Bhat } \\
(2005)\end{array}$ & \multirow{2}{*}{$\begin{array}{l}-1 \\
0 \\
0 \\
\text { II } \\
\theta\end{array}$} & 4.475220544 & 4.584372264 & 4.693523985 & 4.802675705 & 4.911827426 \\
\hline Present Study & & 6.559196214 & 6.71917661 & 6.879157005 & 7.039137401 & 7.199117796 \\
\hline $\begin{array}{l}\text { Shah and Bhat } \\
(2005)\end{array}$ & \multirow{2}{*}{$\begin{array}{l}-1 \\
0 \\
0 \\
11 \\
\theta\end{array}$} & 4.848155589 & 4.966403286 & 5.084650984 & 5.202898681 & 5.321146378 \\
\hline Present Study & & 7.105795899 & 7.279107994 & 7.452420089 & 7.625732184 & 7.799044279 \\
\hline $\begin{array}{l}\text { Shah and Bhat } \\
(2005)\end{array}$ & \multirow{2}{*}{$\begin{array}{l}\overrightarrow{1} \\
0 \\
0 \\
11 \\
\theta\end{array}$} & 5.221090634 & 5.348434308 & 5.475777982 & 5.603121656 & 5.73046533 \\
\hline Present Study & & 7.652395583 & 7.839039378 & 8.025683173 & 8.212326968 & 8.398970762 \\
\hline $\begin{array}{l}\text { Shah and Bhat } \\
(2005)\end{array}$ & \multirow{2}{*}{$\begin{array}{l}\overrightarrow{0} \\
0 \\
0 \\
0 \\
\theta\end{array}$} & 5.59402568 & 5.73046533 & 5.866904981 & 6.003344632 & 6.139784283 \\
\hline Present Study & & 8.198995268 & 8.398970762 & 8.598946257 & 8.798921751 & 8.998897245 \\
\hline
\end{tabular}

Table 1. Profile of $W$ for $\tau$ and $\phi$. 


\begin{tabular}{|c|c|c|c|c|c|c|}
\hline & $\tau$ & 0.1 & 0.2 & 0.3 & 0.4 & 0.5 \\
\hline $\begin{array}{l}\text { Shah and } \\
\text { Bhat } \\
(2005)\end{array}$ & \multirow{2}{*}{$\underset{11}{11}$} & 2.433675036 & 5.887540886 & 14.43968034 & 35.87446052 & 90.16334551 \\
\hline $\begin{array}{l}\text { Present } \\
\text { Study }\end{array}$ & & 3.021753265 & 3.025973857 & 3.029069025 & 3.031342056 & 3.033013821 \\
\hline $\begin{array}{l}\text { Shah and } \\
\text { Bhat } \\
(2005)\end{array}$ & \multirow{2}{*}{$\underset{\substack{0 \\
11}}{102}$} & 2.654918221 & 6.422771876 & 15.75237856 & 39.13577511 & 98.36001329 \\
\hline $\begin{array}{l}\text { Present } \\
\text { Study }\end{array}$ & & 3.296458107 & 3.301062389 & 3.304438936 & 3.306918606 & 3.30874235 \\
\hline $\begin{array}{l}\text { Shah and } \\
\text { Bhat } \\
(2005)\end{array}$ & \multirow{2}{*}{$\underset{11}{11}$} & 2.876161406 & 6.958002865 & 17.06507677 & 42.39708971 & 106.5566811 \\
\hline $\begin{array}{l}\text { Present } \\
\text { Study }\end{array}$ & & 3.57116295 & 3.576150922 & 3.579808848 & 3.582495157 & 3.584470879 \\
\hline $\begin{array}{l}\text { Shah and } \\
\text { Bhat } \\
(2005)\end{array}$ & \multirow{2}{*}{$\underset{\substack{\infty \\
11 \\
1 \infty}}{\stackrel{\infty}{11}}$} & 3.097404592 & 7.493233855 & 18.37777498 & 45.6584043 & 114.7533488 \\
\hline $\begin{array}{l}\text { Present } \\
\text { Study }\end{array}$ & & 3.845867792 & 3.851239454 & 3.855178759 & 3.858071707 & 3.860199408 \\
\hline $\begin{array}{l}\text { Shah and } \\
\text { Bhat } \\
(2005)\end{array}$ & \multirow{2}{*}{$\underset{11}{\stackrel{9}{11}}$} & 3.318647777 & 8.028464845 & 19.69047319 & 48.91971889 & 122.9500166 \\
\hline $\begin{array}{l}\text { Present } \\
\text { Study }\end{array}$ & & 4.120572634 & 4.126327987 & 4.13054867 & 4.133648258 & 4.135927938 \\
\hline
\end{tabular}

Table 2. Profile of $W$ for $\tau$ and $\bar{\beta}$.

A comparison of the present study with the ones mentioned above indicates that the porous and slip effect may influence bearing's performance by selecting the proper range of both the parameters. Perhaps, this could be due to the positive characteristic of the magnetization parameter and $\phi$ (Table 1).

A comparison concerning Table 2 shows that the present study is more preferable for the initial values of magnetization parameters, but if it increases, performance will also be remarkable in the previous study with respect to the upper plate's curvature parameter.

\section{Conclusions}

The load lifting capacity increases when the solid phase's volume concentration and the curvature parameter are increased. From the present theoretical study, the Shliomis model is more effective than Neuringer-Rosenweig's model due to Ferro particles' rotation and moments. The load capacity of annular squeeze film can be made ideal by a proper choice of curvature parameter of the upper plate, slip velocity and porosity. The present study may lead to a new scope toward the optimal performance of the bearing system.

Acknowledgments: Authors gratefully acknowledge the support and constant encouragement of Prof. G. M. Deheri (Department of Mathematics, Sardar Patel University, Vallabh Vidyanagar). We are thankful to the editor and reviewers for their valuable suggestions to improve our work.

\section{References}

Agrawal VK (1986). Magnetic fluid-based porous inclined slider bearing, Wear, 107, 133-139. Bhat MV (2003). Lubrication with a Magnetic fluid. Team Spirit (India) Pvt. Ltd., India. 
Bhat MV, Deheri GM (1991). Porous composite slider bearing lubricated with magnetic fluid, Jap. J. Appl .Phys. 30, 2513-2514.

Bhat MV, Deheri GM (1991). Squeeze film behaviour in porous annular discs lubricated with magnetic fluid, Wear, 151, 123-128.

Bhat MV, Deheri GM (1993). Magnetic-fluid-based squeeze film in curved porous circular discs, J. Magn. Magn. Mater., 127, 159-162.

Bujurke NM, Naduvinamani NB, Basti DP (2007). Effect of surface roughness on the squeeze film lubrication between curved annular plates, Industrial Lubrication and Tribology, 59(4), 178-185.

Deheri GM, Patel SJ (2015). Combined effect of slip velocity and surface roughness on a magnetic squeeze film for a sphere in a spherical seat, Indian Journal of Materials Science, 2015 (ID. 159698), 1-9.

Deheri GM, Patel JR, Patel ND (2016). Shliomis Model Based Ferrofluid Lubrication of a Rough Porous Convex Pad Slider Bearing, Tribology in Industry, 38(1), 46-65.

Gupta J L, Vora KH (1980). Analysis of Squeeze Films between Curved Annular Plates, J. of Lubrication Tech., 102(1), 48-50.

Hanumagowda BN, Savitramma G, Salma A, Noorjahan (2018). Combined effect of PiezoViscous dependency and non-newtonian couple stresses in annular plates squeeze-film characteristics, NCMTA 18 IOP Conf. Series: Journal of Physics: Conf. Series, 1000 (2018), 012083.

Kumar D (1989). Contributions to the Theory of Ferrofluid Lubrication. Ph.D. Thesis, IIT Kanpur, India.

Kumar D, Sinha P, Chandra P (1992). Ferrofluid squeeze film for spherical and conical bearings, Int. J. Engg. Sci., 30(5), 645-656.

Lin JR (2001). Magneto-hydrodynamic squeeze film characteristics between annular disks, Industrial Lubrication and Tribology, 53(2), 66-71.

Mishra SR, Barik M, Dash GC (2018). An analysis of hydrodynamic ferrofluid lubrication of an inclined rough slider bearing, Tribology - Materials, Surfaces \& Interfaces, DOI: 10.1080/17515831.2017.1418280.

Munshi MM, Patel AR, Deheri G (2019). Lubrication of Rough Short Bearing on Shliomis Model by Ferrofluid Considering Viscosity Variation Effect, International Journal of Mathematical, Engineering and Management Sciences, 4(4), 982-997.

Murti PRK (1975). Squeeze film in curved circular plate, J. Lubr. Technol., 97, 650-652.

Patel JR, Deheri GM (2016). Combined Effect of Slip Velocity and Roughness on the Jenkins Model Based Ferrofluid Lubrication of a Curved Rough Annular Squeeze Film, Journal of Applied Fluid Mechanics, 9(2), 855-865.

Patel JR, Deheri G (2013). Shliomis model based magnetic fluid lubrication of a squeeze film in rotating rough curved circular plates, Carib. J. Sci. Tech. 1, 138-150.

Patel JR, Deheri G (2014). Effect of various porous structures on the Shliomis model based ferrofluid lubrication of the film squeezed between rotating rough curved circular plates, FACTA UNIVERSITATIS Series: Mechanical Engineering, 12(3), 305-323.

Patel JR, Deheri GM (2016). Performance of a Ferrofluid Based Rough Parallel Plate Slider Bearing: A Comparison of Three Magnetic Fluid Flow Models, Advances in Tribology, 2016 (ID 8197160): 1-9.

Shah RC, Patel DB (2017). Analysis and comparative study of ferrofluid lubricated circular porous squeeze film-bearings, Proc. IMechE Part J: J Engineering Tribology, 231(11): 14501463.

Shah RC, Bhat MV (2005). Ferrofluid squeeze film between curved annular plates including rotation of magnetic particles, Journal of Engineering Mathematics, 51, 317-324. 
Shah RC, Patel NI, Kataria RC (2016). Some porous squeeze film-bearings using ferrofluid lubricant: A review with contributions, Proc. of the IME Part J: Journal of Engg. Tribology, 230(9): 1157-1171.

Shah RC, Tripathi SR, Bhat MV (2002). Magnetic fluid based squeeze film between porous annular curved plates with the effect of rotational inertia, Pramana-J. Phys., 58, 545-550.

Shah RC, Bhat MV (2004). Ferrofluid squeeze film in a long journal bearing, Tribology International, 37, 441-446.

Shah RC, Bhat MV (2000). Squeeze film based on magnetic fluid in curved porous rotating circular plates, J. Magn. Magn. Mater., 208, 115-119.

Shimpi ME, Deheri GM (2013). Magnetic fluid based squeeze film in rough rotating curved porous annular plates: deformation effect. World Academy of Science, Engineering and Technology International Journal of Mathematical and Computational Sciences, 7(8), 13701080.

Shliomis MI (1972). Effective viscosity of magnetic suspensions, Sov. Phys. JETP, 34, 12911294.

Shukla JB, Kumar D (1987). A theory of ferrofluid lubrication, J. Magn. Magn. Mater., 65, 375378.

Sinha P, Chandra P, Kumar D (1993). Ferrofluid lubrication of cylindrical rollers with cavitation, Acta Mech., 98, 27-38.

Vasanth KR, Hanumagowda BN, Kumar JS (2018). Combined Effect of Piezoviscous Dependency and Non-Newtonian Couple Stress on Squeeze-Film Porous Annular Plate, NCMTA 18, IOP Conf. Series: Journal of Physics: Conf. Series 1000 (2018), 012080. 